\title{
Menopause IUPDATE
}

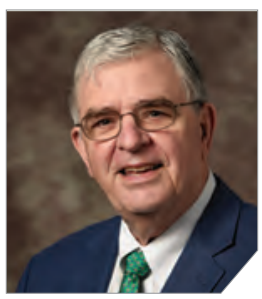

Andrew M. Kaunitz, MD, NCMP

Dr. Kaunitz is Professor and Associate

Chairman, Department of Obstetrics and

Gynecology, University of Florida College of Medicine-Jacksonville; and Medical Director and Director of Menopause and Gynecologic Ultrasound Services, University of Florida Women's Health Specialists at Emerson, Jacksonville. He serves on the OBG MANAGEMENT Board of Editors.

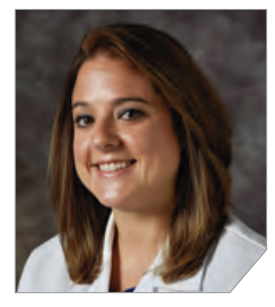

Deanna C. McCullough, MD

Dr. McCullough is Assistant Professor, Department of Obstetrics and Gynecology, University of Florida College of MedicineJacksonville.

Dr. Kaunitz reports receiving grant or research support from Mithra and that the University of Florida receives the funding. Dr. McCullough reports no financial relationships relevant to this article.

Expert perspectives on menopausal hormone therapy and breast cancer risk, nonindicated bilateral salpingo-oophorectomy in pre- and perimenopausal women, benefits of hormone therapy for depression in menopause, and the American Heart Association's statement on the menopause transition and cardiovascular disease risk implications

A mong the studies we review in this Update are a follow-up of the US Women's Health Initiative clinical trials and a large observational study from the United Kingdom, which exlore the impact of different hormone therapies (HTs) on breast cancer risk. We look at the interesting patterns found by authors of a study in Canada that analyzed predictors of unnecessary bilateral salpingo-oophorectomy. In addition, we review a study that investigates whether hormone therapy can be effective, alone or adjunctively, in peri- and postmenopausal women with depression. Finally, Dr. Chrisandra Shufelt and Dr. JoAnn Manson summarize highlights from the recent American Heart Association's scientific statement on the menopause transition and increasing risk factors for cardiovascular disease, and how this period can be viewed as an opportunity to encourage healthy, cardiovascular risk-reducing behaviors.
IN THIS ARTICLE

Nonindicated BSO

page 34

HT for depression page 35

Menopause transition and CVD risk page 40
Chlebowski RT, Anderson GL, Aragaki AK, et al. Association of menopausal hormone therapy with breast cancer incidence and mortality during long-term follow-up of the Women's Health Initiative randomized clinical trials. JAMA. 2020;324:369-380. doi: 10.1001/ jama.2020.9482.

Vinogradova Y, Coupland C, Hippisley-Cox J. Use of hormone replacement therapy and risk of breast cancer: nested case-control studies using the QResearch and CPRD databases. BMJ. 2020;371:m3873. doi: 10.1136/bmj.m3873.

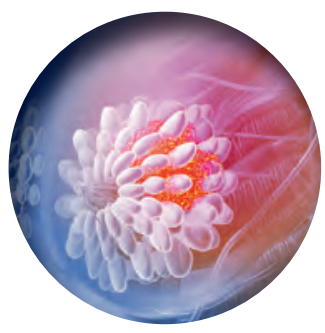

or many menopausal women, the most worrisome concern related to the use of HT is that it might increase breast cancer risk. In the summer and fall of 2020 , 
FAST

TRACK

Forwomen

with previous

hysterectomy

considering ET

for treatment

of bothersome

menopausal

symptoms, the

breast cancer

mortality benefit

documented in

the long-term WHI

analysis could tip the

scales in favor of ET
2 important articles were published that addressed how the use of menopausal HT impacts the risk of breast cancer.

The Women's Health Initiative (WHI) represents the largest and longest-term randomized trial assessing the health impacts of systemic HT. A 2013 WHI report found that with a median of 13 years' cumulative follow-up, estrogen-only HT (ET) reduced the risk for breast cancer while estrogenprogestin therapy (EPT) increased the risk. ${ }^{1}$ In a July 2020 issue of JAMA, WHI investigators analyzed longer-term data (cumulative median follow-up $>20$ years), which allowed assessment of whether these trends (breast cancer incidence) persisted and if they led to changes in mortality from breast cancer. ${ }^{2}$

\section{WHI data on breast cancer risk trends in ET vs EPT users}

In the ET trial, in which Chlebowski and colleagues studied 10,739 women with prior hysterectomy, 238 versus 296 new cases of breast cancer were diagnosed in women in the ET versus placebo groups, respectively (annualized incidence, $0.30 \%$ [ET] vs $0.37 \%$ [placebo]; hazard ratio [HR], $0.78 ; P=.005$ ). ET also was associated with significantly lower mortality from breast cancer: 30 versus 46 deaths (annualized mortality, $0.031 \%$ [ET] vs $0.046 \%$ [placebo]; HR, $0.60 ; P=0.04$ ).

In the EPT trial, which included 16,608 participants with an intact uterus, EPT compared with placebo was associated with significantly elevated risk for incident breast cancer: 584 versus 447 new cases, respectively (annualized incidence, $0.45 \%$ [EPT] vs $0.36 \%$ [placebo]; HR, 1.28 ; $P<.001)$. However, mortality from breast cancer was similar in the EPT and placebo groups: 71 and 53

\section{WHAT THIS EVIDENCE MEANS FOR PRACTICE}

In our practice, for women with an intact uterus who are considering the use of EPT for treatment of bothersome menopausal symptoms, we counsel that long-term use of HT slightly elevates the risk for breast cancer. By contrast, we advise posthysterectomy women with bothersome menopausal symptoms that ET does not appear to increase the risk for breast cancer. deaths (annualized mortality, 0.045\% [EPT] and $0.035 \%$ [placebo]; HR, $1.35 ; P=.11$ ). ${ }^{2}$

For women with previous hysterectomy who are considering initiating or continuing ET for treatment of bothersome menopausal symptoms, the breast cancer mortality benefit documented in this long-term WHI analysis could, as editorialists point out, "tip the scales" in favor of ET. ${ }^{3}$ Furthermore, the mortality benefit raises the possibility that ET could be evaluated as a risk-reduction strategy for selected high-risk menopausal women who have undergone hysterectomy. Although tamoxifen and aromatase inhibitors are approved for breast cancer chemoprophylaxis in high-risk menopausal women, these agents have not been found to lower breast cancer mortality. ${ }^{2}$

\section{UK data analysis and risk for breast cancer in HT users}

In an October 2020 issue of BMJ, Vinogradova and colleagues described their analysis of 2 primary care databases in the United Kingdom that in aggregate included roughly 99,000 women with breast cancer diagnosed between 1998 and 2018 (age range, 50-79; mean age at diagnosis, 63 ; $>95 \%$ White); these were matched with more than 450,000 women without breast cancer (controls). ${ }^{4}$ Analyses were adjusted for smoking, body mass index (BMI), ethnicity, and mammography.

In this study, ever-use of EPT was associated with an adjusted odds ratio (OR) for breast cancer of 1.26 (95\% confidence interval [CI], 1.24-1.29), while ET had an OR of 1.06 (95\% CI, 1.03-1.10). In women aged 50 to 59 who used EPT for 5 years or more, 15 additional breast cancers were diagnosed per 10,000 woman-years; for ET users, the attributable risk was 3 . Although risk rose with longer HT duration, this trend was less evident with ET than EPT.

In addition, the increased risk associated with ET use was less pronounced in women with a BMI greater than $30 \mathrm{~kg} / \mathrm{m}^{2}$. Among EPT users, risks were similar with the progestins medroxyprogesterone acetate (MPA), norethindrone (NET), and 


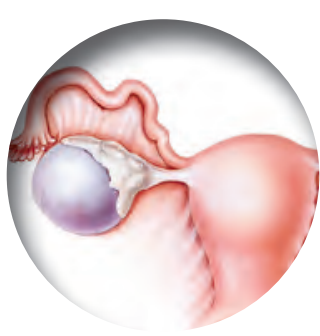

$\overline{\text { FAST }}$

TRACK

BSO at the time of hysterectomy in preor perimenopausal women is associated with increased overall mortality and an increased risk of mortality due to CVD in patients younger than age 45

\section{WHAT THIS EVIDENCE MEANS FOR PRACTICE}

The retrospective study by Wong and colleagues provides the first assessment of Canadian practice patterns with respect to concurrent BSO at the time of hysterectomy. The authors found that, overall, more than two-thirds of BSO procedures were indicated. However, the proportion of BSO that was indicated was higher in teaching hospitals and in surgeries performed by fellowship-trained gynecologists. These important observations underscore the role of clinician education in reducing nonindicated BSO in pre- and perimenopausal women undergoing hysterectomy for benign disease. 2020;28:8-11. doi: 10.1097/GME.0000000000001652.

W compared with risk in never-users. ${ }^{4}$

In this large observational UK study, ET was associated with minimally elevated risk for breast cancer, while in the WHI study, ET reduced the risk for breast cancer. For EPT, the excess risk in both studies was identical. As the authors note, mean BMI in the UK study participants was slightly lower than that in the WHI participants, a distinction that might explain the differing findings with ET use.

\section{Frequency of nonindicated BSO at the time of hysterectomy in pre- and perimenopausal women}

Wong J, Murji A, Sunderji Z, et al. Unnecessary bilateral salpingo-oophorectomy at the time of hysterectomy and potential for ovarian preservation. Menopause. hile prevention of ovarian cancer is an important benefit of bilateral salpingo-oophorectomy (BSO), performing a BSO at the time of hysterectomy in pre- or perimenopausal patients not only will induce surgical menopause but also is associated with significantly increased overall mortality and an increased risk of mortality due to cardiovascular disease in patients younger than age $45 .{ }^{5,6}$ Earlier BSO also has been associated with diabetes, accelerated bone density loss, sexual dysfunction, mood disorders, and decreased cognitive function.

\section{BSO at hysterectomy: How many procedures are not indicated?}

To evaluate the prevalence and predictors of unnecessary BSO at the time of hysterectomy, Wong and colleagues conducted a multicenter retrospective review of hysterectomy procedures completed at 6 Canadian hospitals. ${ }^{8}$ Criteria for unnecessary BSO included age younger than 51 years; benign preoperative diagnosis (other than endometriosis, premenstrual dysphoric disorder, and gender dysphoria); and absence of endometriosis and pelvic adhesions.

A total of 2,656 hysterectomies were performed by 75 surgeons ( 28 fellowship trained and 47 generalists) across 3 community and 3 tertiary care hospitals between 2016 and 2018. At the time of hysterectomy, 749 patients $(28 \%)$ underwent BSO. Of these, 509 women $(68 \%)$ had at least 1 indication for concurrent BSO based on preoperative diagnosis.

Key study findings. Concurrent BSO procedures performed at academic hospitals were more likely to have a preoperative indication compared with BSO performed at community sites $(70 \%$ vs $63 \%$; OR, $1.42 ; 95 \% \mathrm{CI}$, 1.02-1.97; $P=.04)$. BSO was more likely to be indicated when performed by fellowshiptrained surgeons compared with surgeries performed by generalist surgeons $(75 \%$ vs 
63\%; OR, 1.76; 95\% CI, 1.26-2.44, $P=.001$ ). $\mathrm{BSO}$ procedures performed with vaginal hysterectomy were less likely to be indicated (3 of $20,15 \%$ ) when compared with open hysterectomy (74 of 154, 48\%) and laparoscopic hysterectomy ( 432 of $575,75 \%$ ).

Of the patients who lacked a preoperative indication for concomitant BSO, 105 of 239 (43.9\%) were younger than age 51. Overall, $8 \%$ (59 of 749) of patients in the study cohort had an unnecessary BSO based on a combination of preoperative diagnosis, age younger than age 51, and intraoperative factors including absence of endometriosis and adhesions.

\section{HT for menopausal depression: Which patients may benefit?}

Dwyer JB, Aftab A, Radhakrishnan R, et al; APA Council of Research Task Force on Novel Biomarkers and Treatments. Hormonal treatments for major depressive disorder: state of the art. Am J Psychiatry. 2020;177:686705. doi:10.1176/appi.ajp.2020.19080848.

$\mathrm{T}$ he cumulative lifetime prevalence of major depression in US women is $21 \%{ }^{9}$ An increased risk of mood symptoms and major depressive disorder occurs with the cessation of ovarian hormone production during menopause. In a review of both physiology and clinical studies, an American Psychiatric Association task force found support for several hormone-related strategies for treating depression and highlighted the rapidly advancing, but mixed, findings in this field. ${ }^{10}$

Clinical trials that examined mood in peri- and postmenopausal women treated with HT have produced mixed results for a variety of reasons, including differences in psychiatric symptomatology across studies and differences in treatment timing in relation to menopause onset.

\section{HT effectiveness for depression depends on menopausal status}

Five studies included in a meta-analysis by Rubinow and colleagues examined the use of ET and EPT as antidepressant monotherapy in peri- or postmenopausal women with major depression. ${ }^{11}$ Of the 3 higher-quality studies, 2 conducted in perimenopausal women demonstrated the antidepressant efficacy of transdermal estrogen

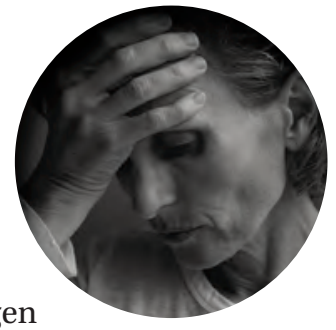
patches compared with placebo. The third study included a mixed population of both peri- and postmenopausal women, and it found that increased estradiol levels (spontaneously occurring or due to ET) were associated with improvement in depression in perimenopausal women but not in postmenopausal women. ${ }^{11}$

ET also has been investigated as a potential adjunctive treatment to selective serotonin reuptake inhibitors (SSRIs). In a retrospective analysis of a multicenter randomized controlled trial of fluoxetine in patients with depression, women who received ET and fluoxetine demonstrated a greater improvement than those who received fluoxetine monotherapy. ${ }^{12}$ One small study that prospectively assessed ET in combination with an antidepressant in postmenopausal women demonstrated no benefit of ET in treating depression..$^{13}$ Another small trial found that while combining transdermal ET with an SSRI accelerated symptom improvement, by the end of the 10-week study, treatment efficacy in the HT plus SSRI group was no greater than that observed in the SSRI-only group. ${ }^{14}$

Nineteen studies included in the metaanalysis by Rubinow and colleagues, which examined mood after ET or EPT treatment in nondepressed women, found little evidence of benefit, particularly in women without other physical symptoms of menopause. ${ }^{11}$

The Kronos Early Estrogen Prevention Study (KEEPS) followed 661 women who 
received either oral estrogen plus progesterone, transdermal estrogen plus progesterone, or placebo over 4 years. ${ }^{15}$ Women with clinical depression were excluded from the study; however, women with mild to moderate mood symptoms who were being treated with an antidepressant were included. Improvements in depressive symptoms and anxiety were observed only in the oral estrogen plus progesterone group compared with the placebo group. ${ }^{15}$

In a study of 172 euthymic peri- and postmenopausal women treated for 12 months with transdermal estrogen plus oral progesterone, investigators found that, unlike postmenopausal women and those in the late perimenopausal transition, only women in the early perimenopausal transition had a lower risk of developing depressive symptoms. ${ }^{16}$

\section{Bottom line}

This complex literature suggests that ET/HT interventions are most likely to be successful when implemented early in the menopausal transition. The clearest indication for the use of HT is for perimenopausal women experiencing

\section{References}

1. Manson JE, Chlebowski RT, Stefanick ML, et al. Menopausal hormone therapy and health outcomes during the intervention and extended poststopping phases of the Women's Health Initiative randomized trials. JAMA. 2013;310:13531368. doi: 10.1001/jama.2013.278040.

2. Chlebowski RT, Anderson GL, Aragaki AK, et al. Association of menopausal hormone therapy with breast cancer incidence and mortality during long-term follow-up of the Women's Health Initiative randomized clinical trials. JAMA. 2020;324:369-380. doi: 10.1001/jama.2020.9482.

3. Minami CA, Freedman RA. Menopausal hormone therapy and long-term breast cancer risk: further data from the Women's Health Initiative trials. JAMA. 2020;324:347-349. doi: 10.1001/jama.2020.9620.

4. Vinogradova Y, Coupland C, Hippisley-Cox J. Use of hormone replacement therapy and risk of breast cancer: nested casecontrol studies using the QResearch and CPRD databases. BMJ. 2020;371:m3873. doi: 10.1136/bmj.m3873.

5. Adelman MR, Sharp HT. Ovarian conservation vs removal at the time of benign hysterectomy. Am J Obstet Gynecol. 2018;218:269-279. doi: 10.1016/j.ajog.2017.07.037.

6. Rivera CM, Grossardt BR, Rhodes DJ, et al. Increased cardio vascular mortality after early bilateral oophorectomy. Menopause. 2009;16:15-23. doi: 10.1097/gme.0b013e31818888f7.

7. Karp NE, Fenner DE, Burgunder-Zdravkovski L, et al. Removal of normal ovaries in women under age 51 at the time of hysterectomy. Am J Obstetr Gynecol. 2015;213:716.e1-6. doi: 10.1016/j.ajog.2015.05.062.

8. Wong J, Murji A, Sunderji Z, et al. Unnecessary bilateral salpingo-oophorectomy at the time of hysterectomy and potential for ovarian preservation. Menopause. 2021;28:8-11. doi: 10.1097/GME.0000000000001652.

9. Kessler RC, McGonagle KA, Swartz M, et al. Sex and depression in the National Comorbidity Survey. I: lifetime preva-

\section{WHAT THIS EVIDENCE MEANS FOR PRACTICE}

A trial of ET/EPT is reasonable in perimenopausal women with depression and classic menopausal symptoms. Use of HT also can be considered either alone or in combination with an SSRI in perimenopausal women with depression who do not have significant classic menopausal symptoms. However, HT is not recommended as prophylaxis against depression in euthymic perimenopausal women. Finally, keep in mind that the use of HT to address mood issues constitutes off-label use.

depression who are also experiencing menopausal symptoms (for example, bothersome hot flashes). There is little evidence that the use of ET/HT in late perimenopausal or postmenopausal women effectively treats depression; accordingly, HT is not recommended for the treatment of mood disorders in this population. The more ambiguous cases are those of perimenopausal women who are depressed but do not have classic vasomotor symptoms; some evidence supports the antidepressant efficacy of HT in this setting. ${ }^{11}$ Although some studies suggest that HT can be effective in preventing depression in perimenopausal women, more evidence is needed. ${ }^{16}$

lence, chronicity, and recurrence. J Affect Disord. 1993;29:8596. doi: 10.1016/0165-0327(93)00026-g.

10. Dwyer JB, Aftab A, Radhakrishnan R, et al; APA Council of Research Task Force on Novel Biomarkers and Treatments. Hormonal treatments for major depressive disorder: state of the art. Am J Psychiatry. 2020;177:686-705. doi:10.1176/appi. ajp.2020.19080848.

11. Rubinow DR, Johnson SL, Schmidt PJ, et al. Efficacy of estradiol in perimenopausal depression: so much promise and so few answers. Depress Anxiety. 2015;32:539-549. doi: 10.1002/ da.22391.

12. Schneider LS, Small GW, Hamilton SH, et al. Estrogen replacement and response to fluoxetine in a multicenter geriatric depression trial. Fluoxetine Collaborative Study Group. Am J Geriatr Psychiatry. 1997;5:97-106.

13. Dias RS, Kerr-Corrêa F, Moreno RA, et al. Efficacy of hormone therapy with and without methyltestosterone augmentation of venlafaxine in the treatment of postmenopausal depression: a double-blind controlled pilot study. Menopause. 2006;13:202-211. doi:10.1097/01.gme.0000198491.34371.9c.

14. Rasgon NL, Dunkin J, Fairbanks L, et al. Estrogen and response to sertraline in postmenopausal women with major depressive disorder: a pilot study. JPsychiatr Res. 2007;41:338343. doi: 10.1016/j.jpsychires.2006.03.009.

15. Gleason CE, Dowling NM, Wharton W, et al. Effects of hormone therapy on cognition and mood in recently postmenopausal women: findings from the randomized, controlled KEEPS-cognitive and affective study. PLoS Med. 2015;12:e1001833. doi: 10.1371/journal.pmed.1001833.

16. Gordon JL, Rubinow DR, Eisenlohr-Moul TA, et al. Efficacy of transdermal estradiol and micronized progesterone in the prevention of depressive symptoms in the menopause transition: a randomized clinical trial. JAMA Psychiatry. 2018;75:149-157. doi:10.1001/jamapsychiatry.2017.3998. 


\section{The menopause transition: A key period for strategizing CVD risk factor reduction}

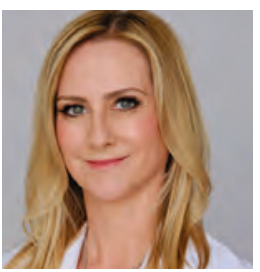

Chrisandra L. Shufelt, MD, MS, NCMP

Dr. Shufelt is Associate Director of the Barbra Streisand Women's Heart Center, Smidt Heart Institute, Cedars-Sinai Medical Center, Los Angeles, California.

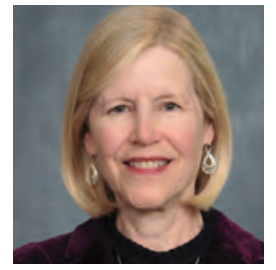

JoAnn E. Manson, MD, DrPH, NCMP

Dr. Manson is Professor of Medicine and the Michael and Lee Bell Professor of Women's Health at Harvard Medical School; Professor in the Department of Epidemiology, Harvard T.H. Chan School of Public Health; and Chief of the Division of Preventive Medicine at Brigham and Women's Hospital, Boston, Massachusetts.

The authors report no financial relationships relevant to this article. Dr. Manson is a coauthor of the AHA Scientific Statement discussed in this article.

n the United States, nearly one-half of a woman's life, on average, will be lived after menopause. For women with natural menopause, the menopause transition (MT) can begin 2 to 7 years before and may extend 1 year past the final menstrual period, which occurs at an average age of 51 years. For women with surgical menopause, the MT occurs abruptly with the sudden loss of endogenous ovarian hormones. Both types of transitions mark a critical time period when reproduction and endogenous sex hormone levels diminish and when cardiovascular disease (CVD) risk factors begin to rise.

The 2020 American Heart Association (AHA) scientific statement, "Menopause transition and cardiovascular disease risk: Implications for timing of early prevention," highlights the MT as a window of opportunity for CVD prevention. ${ }^{1}$

CVD risk factors associated with ovarian aging

In the AHA scientific statement, data from several longitudinal women's health studies were used to identify which CVD risk factor changes during the MT are related to ovarian aging as opposed to chronologic aging. Independent of aging, those associated with reproductive or ovarian aging included an increase in serum total cholesterol, low-density lipoprotein cholesterol (LDL-C), and apolipoprotein B. Changes in high-density lipoprotein cholesterol (HDL-C) particles and function also occur during the MT, which may explain why higher HDL-C levels during the MT and the postmenopausal years are not as cardioprotective as during the premenopausal period.

Changes in body composition and adipose tissue distribution also are associated with ovarian aging, with reduction in muscle mass and lean body mass and an increase in abdominal/visceral fat and subcutaneous adipose tissue. Although these body composition changes reflect ovarian aging, midlife weight gain is more closely related to chronologic aging.

The risk of the metabolic syndrome constellation of risk factors was found to be more closely associated with ovarian aging, whereas changes in blood pressure, insulin, and glucose individually tracked more closely with chronologic aging. Additionally, the AHA statement notes the research that identified several symptoms during the MT-including vasomotor symptoms, sleep disturbance, and depression-as being associated with more adverse CVD risk factor status and with subclinical measures of atherosclerosis. Additional research on the mechanistic basis for these associations is needed.

\section{Chronologic age and type of menopause}

Notably, a woman's age and type of menopause matter with respect to CVD risk. Higher CVD risk is seen in women with premature onset (age < 40 years) or early onset (age $<45$ years) of menopause and in women undergoing surgical menopause (bilateral oophorectomy) before age 45 . In general, menopausal hormone therapy $(\mathrm{HT})$ is recommended for women with premature or early menopause, whether natural or surgical, with continuation through at least the average age of natural menopause. In other women, although not recommended for the express purpose of CVD prevention, menopausal HT is appropriate for the treatment of bothersome vasomotor or other menopausal symptoms, especially when therapy is started before age 60 or within 10 years of menopause among women who are not at elevated risk of CVD.

While the AHA statement suggests that some women who begin estrogen early in menopause may experience reduced coronary heart disease risk, major research gaps remain with regard to HT dose, formulation, route of delivery, and recommended duration of treatment.

An opportunity to promote healthy lifestyle behaviors Translating the AHA's first-of-its-kind scientific statement into clinical practice requires recognition and awareness of the MT as a unique phase in a woman's life associated with myriad changes in CVD risk factors. The statement underscores that the MT is an important time to target behavioral changes to promote CVD risk reduction, including lifestyle modifications in the AHA's Life's Simple 7 components (increased physical activity, smoking cessation, healthy diet, avoidance of weight gain) as well as vigilant control of blood pressure, cholesterol, and glucose levels. The MT is truly a window of opportunity for reinvigorated efforts to lower women's CVD risk.

\section{Reference}

1. El Khoudary SR, Aggarwal B, Beckie TM, et al; American Heart Association Prevention Science Committee of the Council on Epidemiology and Prevention; and Council on Cardiovascular and Stroke Nursing. Menopause transi-

tion and cardiovascular disease risk: implications for timing of early prevention: a scientific statement from the American Heart Association. Circulation. 2020;142:e506-e532. doi: 10.1161/CIR.000000000000912. 